\title{
Correction to: DL-CNN-based approach with image processing techniques for diagnosis of retinal diseases
}

\author{
Akash Tayal $^{1} \cdot$ Jivansha Gupta ${ }^{1,2} \cdot$ Arun Solanki $^{2} \cdot$ Khyati Bisht $^{1} \cdot$ Anand Nayyar $^{3}$ (D) $\cdot$ Mehedi Masud $^{4}$
}

๑) Springer-Verlag GmbH Germany, part of Springer Nature 2021

Correction to: Multimedia Systems

https://doi.org/10.1007/s00530-021-00769-7

In the original publication of the article, the affiliation of the author "Arun Solanki" was numbered incorrectly. It should be 2 instead of 3 .

The original article has been updated with the correct affiliation "Gautam Buddha University, Greater Noida, India”.

Publisher's Note Springer Nature remains neutral with regard to jurisdictional claims in published maps and institutional affiliations.

The original article can be found online at https://doi.org/10.1007/ s00530-021-00769-7.

Anand Nayyar

anandnayyar@duytan.edu.vn

Akash Tayal

akashtayal@yahoo.com

Jivansha Gupta

jivanshagupta@gmail.com

Arun Solanki

ymca.arun@gmail.com

Khyati Bisht

khyatibisht4998@gmail.com

Mehedi Masud

mmasud@tu.edu.sa

1 Indira Gandhi Delhi Technical University for Women,

New Delhi, India

2 Gautam Buddha University, Greater Noida, India

3 Duy Tan University, Da Nang, Viet Nam

4 Department of Computer Science, College of Computers and Information Technology, Taif University,

P.O. Box 11099, Taif 21944, Saudi Arabia 